\title{
Supramolecular Nucleoside-Based Gel: Molecular Dynamics Simulation and Characterization of Its Nanoarchitecture and Self- Assembly Mechanism
}

Maria G. F. Angelerou, ${ }^{\dagger}$ Pim W. J. M. Frederix, ${ }^{\ddagger}{ }^{\circledR 0}$ Matthew Wallace, ${ }^{\S \odot}$ Bin Yang, ${ }^{\dagger}$ Alison Rodger,

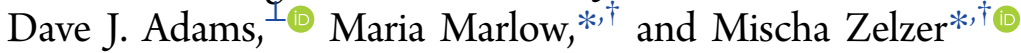

${ }^{\dagger}$ School of Pharmacy, University of Nottingham, Nottingham NG7 2RD, U.K.

${ }^{\ddagger}$ Faculty of Science and Engineering, University of Groningen, Groningen 9747 AG, The Netherlands

${ }^{\S}$ School of Pharmacy, University of East Anglia, Norwich NR4 7TJ, U.K.

"Department of Molecular Sciences, Macquarie University, Sydney, New South Wales 2109, Australia

${ }^{\perp}$ School of Chemistry, University of Glasgow, Glasgow G12 8QQ U.K.

\section{Supporting Information}

\begin{abstract}
Among the diversity of existing supramolecular hydrogels, nucleic acid-based hydrogels are of particular interest for potential drug delivery and tissue engineering applications because of their inherent biocompatibility. Hydrogel performance is directly related to the nanostructure and the self-assembly mechanism of the material, an aspect that is not well-understood for nucleic acid-based hydrogels in general and has not yet been explored for cytosine-based hydrogels in particular. Herein, we use a broad range of experimental characterization techniques along with molecular dynamics (MD) simulation to demonstrate the complementarity and applicability of both approaches for nucleic acidbased gelators in general and propose the self-assembly mechanism for a novel supramolecular gelator, $N^{4}$-octanoyl-2'-deoxycytidine. The experimental data and the MD simulation are in complete agreement with each other and demonstrate the formation of a hydrophobic core within the fibrillar structures of these mainly water-containing materials. The characterization of the distinct duality of environments in this cytidine-based gel will form the basis for further encapsulation of both small hydrophobic drugs and biopharmaceuticals (proteins and nucleic acids) for drug delivery and tissue engineering applications.

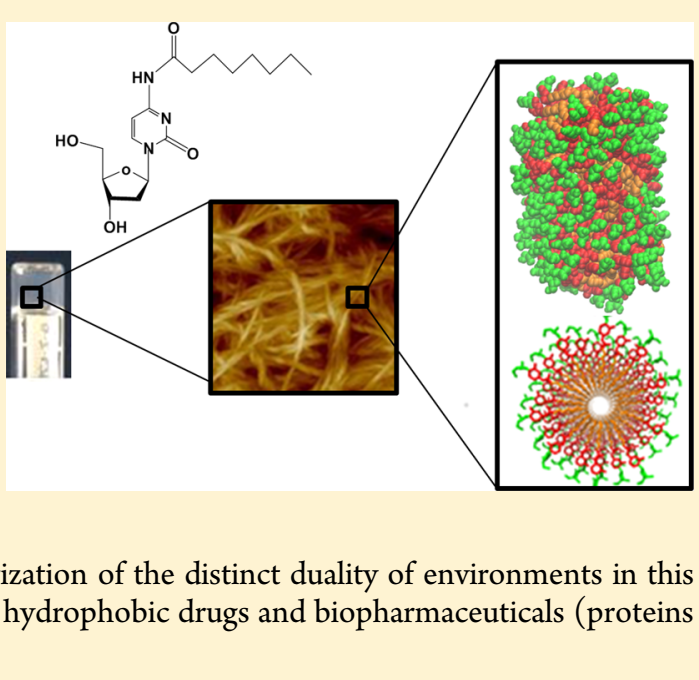

\section{INTRODUCTION}

In the last 10 years, there has been increasing interest in supramolecular gels because of their potential applications as drug delivery systems, sensors, and tissue engineering scaffolds. ${ }^{1-7}$ Derivatives of oligopeptides ${ }^{8,9}$ and nucleic acids, that is, nucleobases, nucleotides, or nucleosides, ${ }^{10,11}$ have been extensively investigated as supramolecular gelators for biological applications because of their inherent biocompatibility. Nucleic acid-based gelators, in particular, are attractive because they are expected to have improved stability toward enzymatic degradation compared to peptide-based gelators.

Nucleic acid-based gels are increasingly finding their way into applications in drug delivery. They can be promising injectable delivery systems ${ }^{12}$ of small therapeutic molecules as well as macromolecules such as proteins and nucleic acids. ${ }^{4,13,14}$

For example, guanosine-based gels have been used to deliver small drug molecules in a controlled way. A $5^{\prime}$-deoxy- $5^{\prime}$ iodoguanosine gel was used to release antivirals, ${ }^{15}$ and a guanosine-5-hydrazide gel was able to incorporate different pharmacologically active molecules including acyclovir, vitamin
C, and vancomycin. ${ }^{16}$ Furthermore, thymidine-based gels have been reported in drug delivery systems for the release of macromolecules. Kaplan et al. presented a thymidine-based mechanoresponsive hydrogel for the delivery of antibodies. ${ }^{13}$ Ramin et al. demonstrated the sustained release of both a large and a small molecule in vivo for the first time. ${ }^{4}$ Maisani et al. have also successfully demonstrated implantation of a thymidine-based composite hydrogel as a scaffold for bone tissue engineering. ${ }^{17}$ Notably, among the existing examples of nucleic acid-based gels, guanine and cytosine derivatives are underrepresented and poorly investigated despite their attractiveness due to the possibility to access G-quadruplexes or i-motifs, ordered structures formed specifically by guanineand cytosine-rich nucleic acid sequences, respectively, that may provide cavities to host payloads in a gel. ${ }^{11}$

Received: February 26, 2018

Revised: $\quad$ May 9, 2018

Published: May 14, 2018 
We have recently introduced a cytosine-based gelator, a fatty acid bound to 2'-deoxycytidine, and reported the bulk mechanical properties of the resulting gels. ${ }^{18}$ Importantly, we also demonstrated that the mechanical properties of the gel are affected by the surface that is in contact with the gelator solution during gelation. ${ }^{19}$ Surface-assisted self-assembly has been recognized as a largely unexplored but significant factor in self-assembly and is receiving increasing attention, ${ }^{20}$ not least because of the implications involved when formulating gels in situ or in the presence of other components such as macromolecules or particles.

To enable a rational design of a class of gelators as well as the investigation and understanding of parameters that influence self-assembly and ultimately gel properties and application, it is essential to elucidate the self-assembly mechanism. In contrast to peptide-based gelators for which the self-assembly mechanism has been extensively explored, ${ }^{9,21}$ a detailed, experimentally supported understanding of the self-assembling mechanism of nucleic acid-based gelators is lacking.

To date, evaluation of nucleic acid self-assembly typically plays an ancillary role where one or two techniques are used to investigate a specific component of the gelator. Barthélémy and coworkers reported small angle X-ray scattering (SAXS) data for an uracil-based gelator, indicating strongly aggregated assemblies that were observed as fibers under transmission electron microscopy (TEM). ${ }^{22}$ As an organogel, the same gelator displayed repeat periods $(4.6 \mathrm{~nm})$ that the authors interpreted to indicate orientation of the hydrophobic part of the gelator toward the organic solvent. In a later study, Barthélémy et al. used the mismatch in the fiber diameter determined by SAXS and the length of the gelator obtained by CPK modeling to propose an interdigitated organization of a thymine based gelator. ${ }^{7}$ Iwaura et al. used X-ray diffraction data of freeze-dried thymine-based gels to argue that the gelator headgroup bends to expose hydroxyl groups to the outside of the fibers. ${ }^{23}$ Temperature-dependent transmittance and circular dichroism (CD) measurements have also been used to determine the gelation temperature of thymine-, adenine-, and uracil-based gels. ${ }^{23,24}$ Banerjee et al. investigated the effect that different functional groups of self-assembling pyrimidine analogues can have on the final fibrillary network. ${ }^{25}$ Roviello et al. used UV, CD, and light scattering to investigate the formation of supramolecular networks of two thymidyl dipeptides and assess their interactions with biomolecules. ${ }^{26}$ To the best of our knowledge, no detailed investigation is currently available on the self-assembly organization or mechanism of cytosine-based gelators. Moreover, while some data on other nucleic acid-based gelators exist, a comprehensive experimental and theoretical description of the contribution of all components in an amphiphilic nucleobase gelator has not yet been reported.

In this work, we systematically explore the self-assembly mechanism of the deoxycytidine derivative $N^{4}$-octanoyl- $2^{\prime}$ deoxycytidine. This gelator is the only member of a class of thermoresponsive cytidine-based gelators developed by our group $^{27}$ that forms a self-healing hydrogel, ${ }^{18}$ and it has been reported as a promising candidate for applications in drug delivery (e.g., as depots for controlled release via gel erosion and diffusion) and tissue engineering along with the macromolecular properties of the gel (e.g., rheology). ${ }^{18}$

Herein, we use a range of experimental approaches to identify the contribution of the different parts of the gelator to the self-assembly process to present the first complete experimental elucidation of the self-assembled organization of a nucleic acid-based gelator. Molecular dynamics (MD) simulations are ideally suited to probe the initial stages of assembly as well as the dimensions and spectroscopic properties of fully assembled nanostructures. ${ }^{28-30}$ They have been successfully applied to monolayers and bilayers of nucleolipids and their hybridization with single-stranded DNA. ${ }^{31,32}$ Taking advantage of this, here, we further combine our experimental dataset with $\mathrm{MD}$ simulations to demonstrate for the first time the complementary match of experimental and theoretical data for a nucleoside gelator. This combined approach enables us to propose a detailed, comprehensive model of the self-assembly of this cytidine-based gelator and pave the way for a rational design of this class of gelators.

\section{RESULTS AND DISCUSSION}

Gels are formed from $N^{4}$-octanoyl-2'-deoxycytidine using a solvent mixture of 20:80 v/v \% ethanol/water. In the mixed solvent system used, the gelator forms a gel composed of tubular fibers (Figure S1, Supporting Information). While the gelator is able to form gels in water only ${ }^{18}$ and does not form gels in organic solvents (e.g., methanol), the addition of ethanol in the solvent improves the solubility of the gelator, facilitating the preparation process and gives a transparent gel ideal for spectroscopy studies.

Self-Assembly Induces Gelator Fluorescence. For gelators that contain fluorescent moieties that are involved in the self-assembly process (e.g., Fmoc- or naphthyl-conjugated peptides), fluorescence spectroscopy has been widely used to study their self-assembly processes. ${ }^{8,33-36}$ Unmodified nucleosides are not inherently fluorescent as shown for the example of 2 -deoxycytidine in Figure 1A. In contrast, $N^{4}$-octanoyl-2'deoxycytidine, the modified $2^{\prime}$-deoxycytidine derivative used

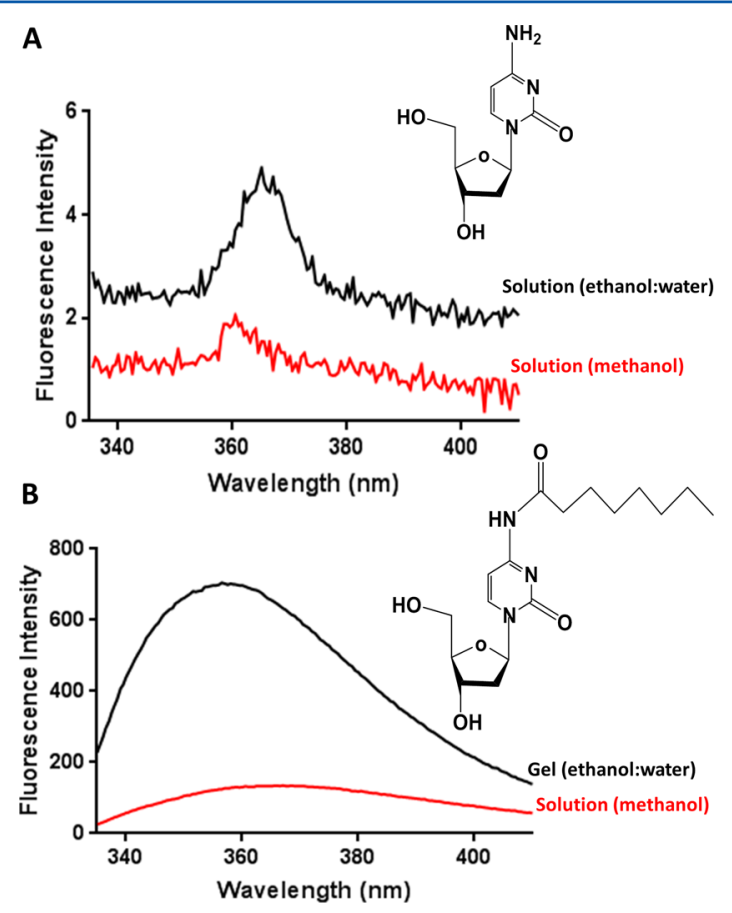

Figure 1. Fluorescence emission spectra of (A) 2'-deoxycytidine and (B) $N^{4}$-octanoyl-2'-deoxycytidine at a concentration of $14 \mathrm{mM}$ in either $20: 80 \mathrm{v} / \mathrm{v} \%$ ethanol/water (black trace) or methanol (red trace) upon excitation at $326 \mathrm{~nm}$. 
here, displays weak fluorescence $\left(\lambda_{\mathrm{Ex}}=326 \mathrm{~nm}, \lambda_{\mathrm{Em}}=367 \mathrm{~nm}\right.$, see Figure S2 for excitation and emission spectra) in methanol, where the molecule is fully soluble (Figure 1B), suggesting that fluorescence might be a useful tool to monitor self-assembly of this nucleoside gelator into structures where the aromatic moiety is protected from water.

In a mixture of $20: 80 \mathrm{v} / \mathrm{v} \%$ ethanol/water, where $N^{4}$ octanoyl-2'-deoxycytidine forms a supramolecular gel, ${ }^{18}$ the intrinsic fluorescence indeed shows a marked increase compared to $N^{4}$-octanoyl-2'-deoxycytidine in methanol and $2^{\prime}$-deoxycytidine in ethanol/water (Figure 1). This suggests that the self-assembled environment enhances the fluorescence of $N^{4}$-octanoyl-2'-deoxycytidine, leading to aggregation-induced emission, ${ }^{5}$ likely because of an increase in the $\pi-\pi$ interactions of the aromatic rings in the nucleobase that protects the molecule's fluorescent chromophore from solventinduced quenching and leads to a strong emission at $357 \mathrm{~nm}$ (Figure 1B, black trace). Because of the significant difference in the fluorescence intensity between the solution and the gel sample, we postulate that the increase in the fluorescence intensity is more likely to be related to the self-assembled state affecting quenching rather than any solvent-induced changes in molecular fluorescence.

To gain further understanding of the arrangement of the nucleobases in the gel, $\mathrm{CD}$ experiments were performed. Aromatic nucleobase monomers are achiral molecules that become $\mathrm{CD}$-active because of their proximity to the chiral sugar. ${ }^{37}$ As with double-stranded DNA, when the chiral nucleosides stack, they gain further $\mathrm{CD}$ intensity if the assembly is helical. As explained in detail in the Supporting Information, $\mathrm{CD}$ and linear dichroism data confirmed the $\pi-\pi$ stacking interactions of the nucleobases in the gel state.

Hydrophobic Domains are Formed in the Gel Fibers. For nucleoside-based amphiphiles, limited evidence has been provided to describe how their hydrophobic parts interact with each other. The presence of hydrophobic environments in the fibers of a cholic acid-based gel has been confirmed in the past through fluorescence by incorporating 8-anilinonaphthalene-1sulfonic acid into the supramolecular system. ${ }^{38}$ Nile red, a poorly water-soluble dye that dissolves and fluoresces strongly in hydrophobic environments ${ }^{39}$ was therefore added to the gels to investigate if hydrophobic pockets are present in the selfassembled structures. After excitation of the gel containing Nile red at $540 \mathrm{~nm}$, strong fluorescence at $630 \mathrm{~nm}$ was obtained (Figure 2A, red trace) that was absent in the control sample (Figure 2A, black trace) where a lower intensity peak at $660 \mathrm{~nm}$ was observed. Super resolution fluorescence microscopy showed that the fluorescence signal is spatially arranged in fiber-like structures (Figure 2B). These data clearly demonstrate the presence of a well-defined hydrophobic environment within the fiber structure.

Effect of Temperature on the Self-Assembly. As noted above, two different fluorescence signals (the gelator's intrinsic fluorescence and the fluorescence of an incorporated dye) can be related to $\pi-\pi$ stacking-related exclusion of solvent and the association of the dye with the hydrophobic part of the gelator, respectively. By monitoring the effect of the temperature on the two different fluorescence signals, we can understand how the hydrophobic interactions contribute to the self-assembly formation and how they relate to each other.

The change in fluorescence emission intensities of the gelator itself with temperature $\left(\lambda_{\mathrm{Ex}}=326 \mathrm{~nm}\right)$ is presented in Figure 3. The intrinsic gel fluorescence is high at room temperature but
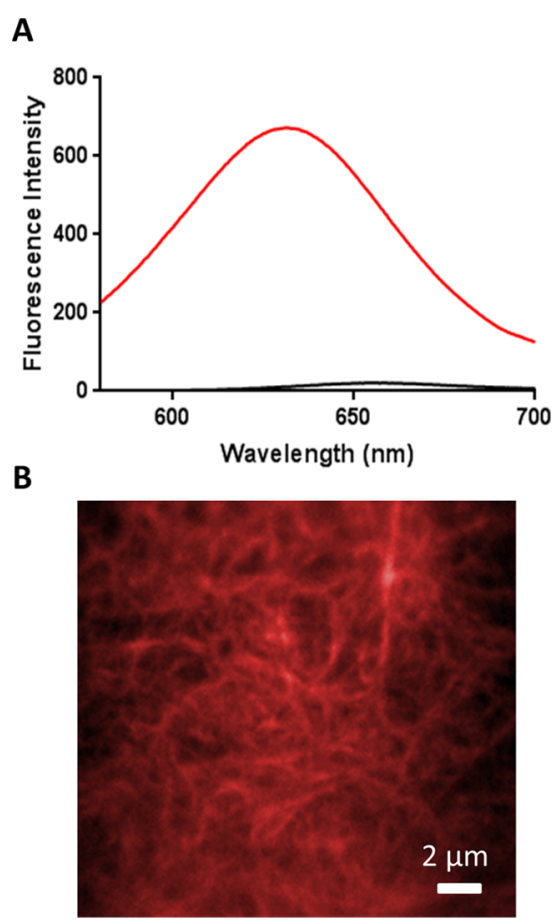

Figure 2. (A) Fluorescence emission spectra $\left(\lambda_{\mathrm{Ex}}=540 \mathrm{~nm}\right)$ of Nile red (approximately $0.1 \mathrm{mM}$ ) in ethanol/water $(20: 80 \mathrm{v} / \mathrm{v} \%)$ (black trace) and the gel after the incorporation of Nile red in ethanol/water $(20: 80 \mathrm{v} / \mathrm{v} \%)$ (red trace). (B) Super resolution fluorescence microscopy image of gels after the incorporation of Nile red.

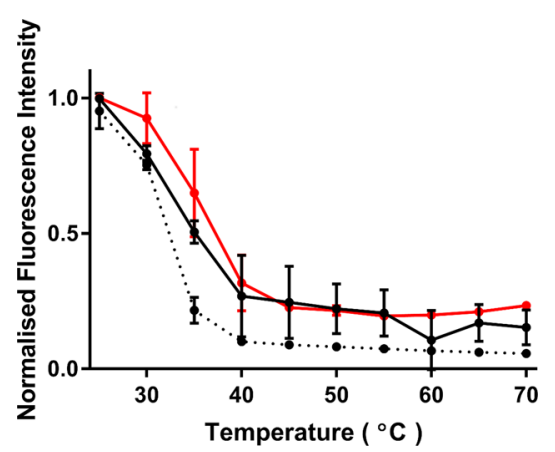

Figure 3. Temperature-dependent change in fluorescence emission intensities of the $N^{4}$-octanoyl-2'-deoxycytidine gelator during the gelsol transition (heating up from 25 to $70{ }^{\circ} \mathrm{C}$ ). Gels in ethanol/water $(20: 80 \mathrm{v} / \mathrm{v} \%)$ after excitation at $326 \mathrm{~nm}$ and emission at $382 \mathrm{~nm}$ (inherent gelator fluorescence, dashed trace) and gels after the incorporation of Nile red in ethanol/water $(20: 80 \mathrm{v} / \mathrm{v} \%)$ after excitation at $540 \mathrm{~nm}$ and emission at $625 \mathrm{~nm}$ (Nile red fluorescence, red trace) and after excitation at $326 \mathrm{~nm}$ and emission at $360 \mathrm{~nm}$ (inherent fluorescence in presence of Nile red, black trace). The intensities were normalized to the highest observed value in each condition. The bars represent the standard deviations (number of repeats $N=3$ ).

drops drastically as the temperature increases and flattens out after $40{ }^{\circ} \mathrm{C}$. Visual inspection (using the "vial inversion test" ${ }^{\prime 0}$ ) showed that this temperature coincides with the transition of the gel into a solution and confirms that the intrinsic gelator fluorescence at room temperature is related to the presence of self-assembled structures. A control experiment monitoring the change of the fluorescence signal over time at a constant temperature (Supporting Information Figure S9) demonstrates that the differences observed in Figure 3 cannot be explained by 
thermal instability of the gel and are indeed related to the change in temperature. Fluorescence therefore also provides a direct route to study the behavior of the nucleobase in the selfassembly process and supports the hypothesis that during selfassembly, $\pi-\pi$ stacking of the nucleobases occurs.

The same experiment was conducted on gels containing Nile red (Figure 3; control experiment of Nile red alone is presented in Figure S10, Supporting Information), after excitation at two different wavelengths: $326 \mathrm{~nm}$ (intrinsic fluorescence from the gelator) and $540 \mathrm{~nm}$ (fluorescence from Nile red). There is a small shift in the transition temperature between the gel containing a dye (black trace) and the plain gel (dashed trace), both after excitation at $326 \mathrm{~nm}$, even at this low concentration of dye, suggesting that the dye is having a stabilizing effect on the gel structure. When the gels containing the dye were excited at the two different wavelengths, the data matched closely, indicating that Nile red is interacting with the hydrophobic, aromatic part of the gelator.

NMR spectroscopy has been used extensively to elucidate supramolecular gel formation ${ }^{41-43}$ and hence was used here to further study the effect of temperature on self-assembly. In the gel state, the resonances of the gelators are very broad because of the low mobility of the gelators in the self-assembled fibers. The assembled gelator's resonances are therefore not visible by solution-state ${ }^{1} \mathrm{H}$ NMR. Thus, the fraction of the mobile gelator in the solution in a sample can be determined by integration of the ${ }^{1} \mathrm{H}$ resonances of the gelator against an internal standard. This approach allows quantification of the relative amount of the gelator in solution versus the amount of the gelator in the assembled fibers. ${ }^{4-46}$ In addition, saturation transfer difference (STD) NMR has been proven useful as a tool to study the exchange between assembled gelator molecules and mobile molecules in solution. ${ }^{47-49}$ In this technique, the very broad ${ }^{1} \mathrm{H}$ resonances of the gel fibers are selectively saturated with lowpower radiofrequency irradiation. Molecules in exchange with the gel fibers receive some of this saturation and therefore exhibit weaker ${ }^{1} \mathrm{H}$ NMR resonances in the ${ }^{1} \mathrm{H}$ NMR spectrum recorded immediately after saturation compared to nonsaturated samples.

In Figure 4, NMR integrals and STDs of proton signals for gel samples are plotted as a function of temperature. Protons have been assigned and grouped into aromatic, aliphatic, and deoxyribose (sugar) (Supporting Information, Figure S11). STD effects are quantified as described in Supporting Information, Figure S11 and eq S1. A strong STD effect indicates the existence of a significant exchange between free

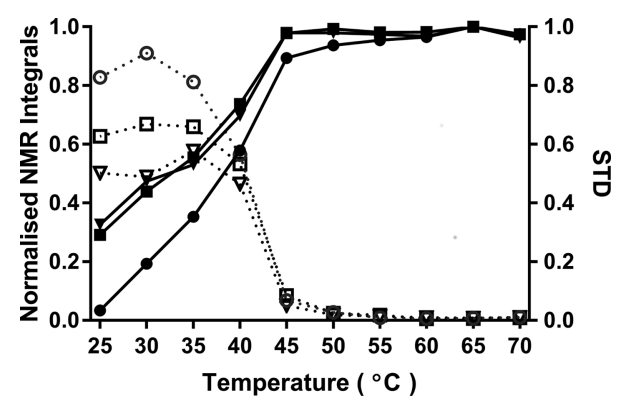

Figure 4. Plots of ${ }^{1} \mathrm{H}$ NMR integrals measured on $N^{4}$-octanoyl-2'deoxycytidine gels against temperature. STDs are also plotted (dashed traces). Trimethylsilylpropanoic acid (TSP) was used as the reference for integration. Different groups of the gelator's protons are presented; aromatic (circles), deoxyribose (triangles), and aliphatic (squares). and assembled gelator molecules. ${ }^{47,49}$ Between 25 and $45{ }^{\circ} \mathrm{C}$, strong STD effects are observed while integration of the gelator resonances indicates that only a fraction of the gelator molecules are NMR-visible. A significant population of the gelator is therefore aggregated at these temperatures, while a degree of exchange exists between the assembled gelators and those in solution. Above $55{ }^{\circ} \mathrm{C}$, no STD effects are observed and no increases in the NMR integrals with temperature relative to an internal standard are discernible. The gelators, therefore, have a high degree of mobility at these temperatures with no NMR-invisible assemblies present. Below $35^{\circ} \mathrm{C}$, the STD effects are saturated and there is no clear change with temperature. $^{44}$

As discussed above, the gelator's fluorescence is directly related to the $\pi-\pi$ stacking, whereas the NMR data shed light into the mobility of the gelator's molecules. The discrepancy in the dissociation temperatures observed between the fluorescence $\left(40^{\circ} \mathrm{C}\right)$ and $\mathrm{NMR}\left(45^{\circ} \mathrm{C}\right)$ may indicate that even if the $\pi-\pi$ interactions become weaker (fluorescence data), the gelator molecules are still, to some extent, assembled (NMR data).

As the temperature increases, the NMR integrals increase until they plateau, while the STD effects decrease to zero. The difference between the initial NMR integrals (at temperatures $<35{ }^{\circ} \mathrm{C}$ ) and the integrals at higher temperatures $\left(>55{ }^{\circ} \mathrm{C}\right)$ is greater for the aromatic protons than the aliphatic resonances. This observation indicates that at lower temperatures $\left(<35^{\circ} \mathrm{C}\right)$, the aromatic group has a much lower mobility in the selfassembled fibers than at the alkyl chain. ${ }^{50}$ At lower temperatures, the integrals of the deoxyribose resonances overlap slightly with those of the aliphatic tail (Supporting Information, Figure S11), thus precluding a detailed comparison of the integrals of the deoxyribose resonances with those of the aromatic and aliphatic resonances. The alkyl chain resonances chosen for this integration did not overlap significantly with the deoxyribose resonances.

Tracking Self-Assembly through Fluorescence Properties. The fact that the fluorescence can be associated with the hydrophobic part of the molecule can give us valuable information on how the self-assembly process progresses during gel formation. ${ }^{51}$ Tracking these two fluorescence signals (the gelator's intrinsic fluorescence and the dye's fluorescence) over time can provide a direct way to measure the gelation time and the gel's stability with time.

Gelator solutions (with and without the dye), after incubation at $60{ }^{\circ} \mathrm{C}$, were directly pipetted into a cuvette, and the intensity of the fluorescence was measured over time (Figure 5). For the gel (without the dye, red data), the fluorescence is of low intensity until the onset of the gel formation, where the signal increases rapidly and levels off again as the gel forms. However, two small peaks are present at early time points, suggesting the formation of initial oligomers, presumably related to early stage $\pi-\pi$ interactions.

With respect to the gel containing Nile red, we excited at two different wavelengths, $326 \mathrm{~nm}\left(\lambda_{\mathrm{Em}}=382 \mathrm{~nm}\right.$ for the gelator's intrinsic fluorescence) and $540 \mathrm{~nm}\left(\lambda_{\mathrm{Em}}=625 \mathrm{~nm}\right.$ for Nile red fluorescence). There is strong fluorescence emission at $2 \mathrm{~min}$ in both cases (black data at $540 \mathrm{~nm}$ and blue data at $326 \mathrm{~nm}$ ), probably because of the temporary generation of localized hydrophobic environments that decreases and increases again, leveling off when the gel is fully formed at $7 \mathrm{~min}$. This suggests a complex process of initial assembly and reorganization into stable extended structures. Even if accurate time lengths cannot 


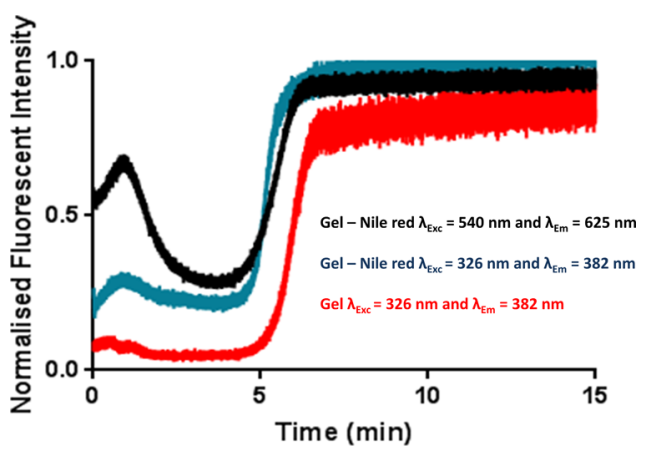

Figure 5. Gel formation; normalized fluorescence emission intensity vs time during gel formation (cooling down process from 60 to $25^{\circ} \mathrm{C}$ ). Fluorescence of gels in ethanol/water $(20: 80 \mathrm{v} / \mathrm{v} \%)$ upon excitation at $326 \mathrm{~nm}$ and emission at $382 \mathrm{~nm}$ [with (blue trace) and without nile red (red trace)] and fluorescence of gels after the incorporation of Nile red in ethanol/water upon excitation at $540 \mathrm{~nm}$ and emission at 625 $\mathrm{nm}$ (black trace). The intensities were normalized to the highest observed value.

be measured because of experimental limitations (there is approximately $30 \mathrm{~s}$ of uncertainty because of variations between sample preparation and starting the measurements), the three data sets almost overlay (the data where the dye is present are slightly shifted to earlier times, further indicating its role in stabilizing the gels). The fact that all the data start leveling out at approximately the same time point $(7 \mathrm{~min})$ confirms that the turning point in the fluorescence traces corresponds to the time required for gelation. Visual observations confirm the fact that the gel is formed (and becomes self-supporting within the first $10 \mathrm{~min}) .{ }^{18}$ Gelation time is usually measured through rheology measurements that involve the application of strain., ${ }^{9,52}$ Measuring the gelation time through fluorescent emission is a noninvasive way to determine gelation time in this system, which gives an advantage because no strain is applied during the gel formation, and so no mechanical perturbation to the structure formed.

Probing the Nanostructures. To gain further insight into the local packing of the molecules after assembly, $N^{4}$-octanoyl2 -deoxycytidine xerogels were prepared and analyzed by X-ray powder diffraction (pXRD) (Figure 6). Four main peaks can be identified. The first two sharp peaks at $2 \theta 5.19^{\circ} / 17.0 \AA$ and $7.86^{\circ} / 11.2 \AA$ (ratio $2: 3$ ) could indicate a lamellar structure with a d-spacing of $34 \AA$ (ratio 1:2:3, suggesting the diameter of the fiber and the dimension of a single molecule to be approximately $17 \AA$ ). However, data collection at lower $2 \theta$ to

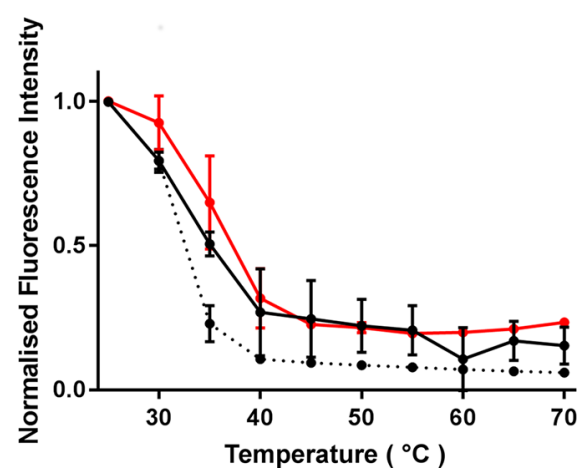

Figure 6. pXRD data obtained from xerogels of $N^{4}$-octanoyl-2'deoxycytidine. confirm assignment of the structures was not possible because of instrumental constraints, and hence, unambiguous identification of the lamellar structure was not possible. In addition to this, drying-induced artefacts such as fiber aggregation could affect the results. ${ }^{53}$

Two broad peaks at $20.5^{\circ}$ and $26.0^{\circ}$ (and a shoulder at $\sim 14.4^{\circ}$ ) dominate the spectrum in Figure 6, corresponding to spacings of 4.3 and $3.4 \AA$ (and $6.1 \AA$ ). The gelator molecules self-assemble into loose, flexible structures, held together through $\pi-\pi$ interactions and hydrogen bonds. Considering this, these two broad peaks are attributed to the different spacings of the nucleobases along the fiber axis, supported by different $\mathrm{N}-\mathrm{H}-\mathrm{O}$ bonds formed between the gelator molecules.

Because pXRD data did not conclusively allow estimation of the fiber diameter and could only be performed on dried samples, small angle neutron scattering (SANS) data for the wet gels were acquired to estimate the diameters of the fibers formed. The data were fitted to a flexible cylinder model with a polydisperse cross section. The results are presented in Figure S12, Supporting Information. All parameters for the best fit are shown in Table S1. The fitting result indicates that the radius of the main unit of which the gel is composed is about $6.88 \pm 0.05$ $\mathrm{nm}$, giving a fiber diameter of approximately $14 \mathrm{~nm}$. As the calculated maximum length of an individual gelator molecule (1.7 nm, see the MD Simulation section below) is an order of magnitude smaller than the measured fiber diameter, it is reasonable to assume that SANS did not measure individual fibers but that the gel architecture is complex and composed of multiple fibers. Hierarchical self-assembly into structures of increasing complexity has been clearly demonstrated in the past for nucleobase-based amphiphiles. Bombelli et al. reported the self-assembly of 1,2-dilauroyl-phosphatidyl-uridine into cylindrical aggregates that under suitable conditions would form giant worm-like micelles entangled into a network. ${ }^{54}$ Moreau et al. demonstrated how the hexagonal packing of helical structures formed by 1,2-dipalmitoyluridinophosphocholine could result in fibers.

Probing the Self-Assembly through MD. To understand the mechanism of the self-assembly on the nanoscale in more detail, we performed $\mathrm{MD}$ simulations of gelator molecules in 20:80 v/v \% ethanol/water. For the self-assembly simulations, 50 or 150 molecules of the gelator were randomly placed in a small periodic box of water and ethanol (Figure 7A), and the simulations were run for 150 and $350 \mathrm{~ns}$, respectively. In the low concentration simulation, gelator molecules formed small aggregates within $5 \mathrm{~ns}$, where the molecules were preferentially organized in a parallel fashion, maximizing aliphatic-aliphatic and parallel-displaced $\pi-\pi$ contacts (Figure $7 \mathrm{~B}$ ). Note that the solvent distribution around the aggregates was not homogeneous: aliphatic tails were mainly solvated by ethanol, while the cytosine bases were mainly solvated by water, and the deoxyribose group environment was well-represented by the overall solvent composition. In the final assembly, ethanol was observed in the periphery of the fiber (nucleobases) but did not penetrate to the hydrophobic core. The aggregate size continuously increased by creating small micelle-like assemblies (Figure $7 \mathrm{C}$ ). These assemblies had a transient nature, often reordering or dissociating until all molecules were in a single, flexible aggregate resembling a micelle (Figure 7D). When the concentration of the gelator molecules was tripled, a similar assembly path was observed, but it resulted in a onedimensional assembly that stretched across the periodic 

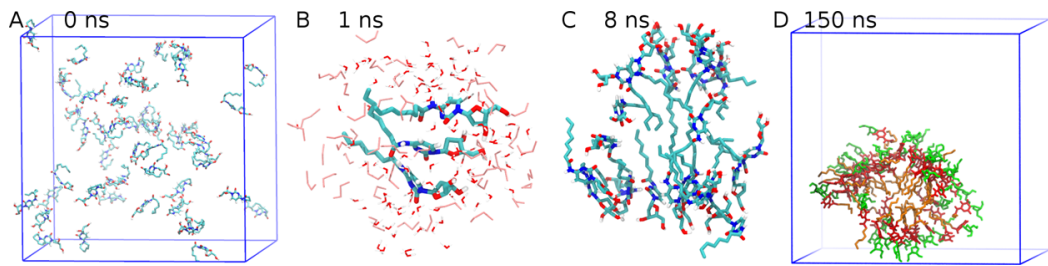

Figure 7. Snapshots from an MD simulation of the self-assembly of 50 gelator molecules in $20: 80 \mathrm{v} / \mathrm{v} \%$ ethanol/water. (A) Starting point of randomly dispersed gelator molecules. Periodic boundary conditions are indicated by the blue cube. Solvent is omitted for clarity. (B) From 0 to 5 ns, small aggregates of parallel-aligned gelator molecules were observed. Note that the hydrophobic tail is preferentially solvated by ethanol (pink), while the polar nucleobases are stacking and solvated by water. (C) Transient micellar structures are observed from 5 to $150 \mathrm{~ns}$. (D) Endpoint of a 150 ns simulation displaying a micellar assembly. Green: deoxyribose red: nucleobase, orange: aliphatic tail.

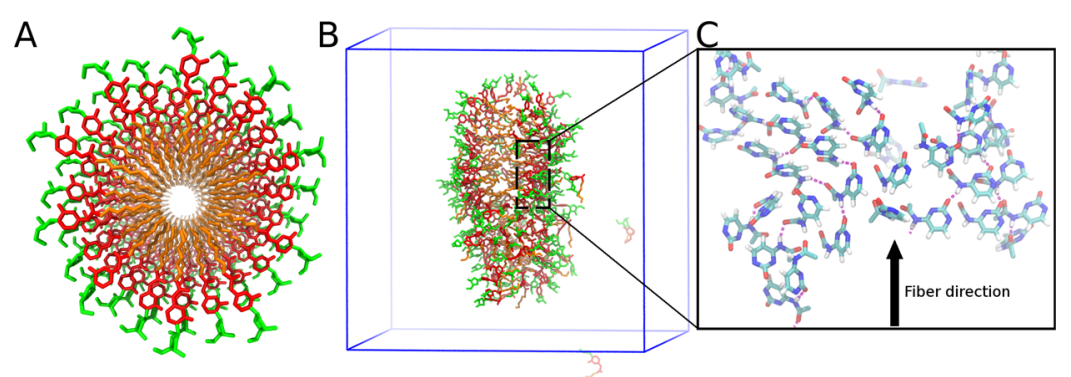

Figure 8. Snapshots from an MD simulation of 160 pre-ordered gelator molecules in ethanol/water. (A) Chiral starting structure. Green: pentose, red: nucleobase, orange: aliphatic tail. (B) Final structure after $50 \mathrm{~ns}$. Periodic boundary conditions are indicated by the blue cube. Solvent is omitted for clarity. (C) Expansion of only the cytosine bases in the fiber indicating H-bonding (in purple) and parallel-displaced $\pi-\pi$-stacking.

boundary of the simulation box (Figure S13). This fiber or worm-like micelle still exhibited a large degree of heterogeneity though, which could be caused by the limited simulation time or the inherent flexible nature of the fibers as observed in the experiments.

To confirm the stability of the fibrous structures and also gain a better insight into the characteristics of the interactions between molecules, we designed a nanostructure by preordering different numbers of gelator molecules into a fibrous structure in a chiral fashion (see Figure 8A), based on common patterns observed in the spontaneous assembly simulation. $\mathrm{MD}$ simulations for $150 \mathrm{~ns}$ revealed that flattening of the fiber was observed, but the base-base stacking, hydrogen bonding, and the tail-tail interactions remained mostly stable near the middle of the fiber (Figure 8B,C).

As the X-ray data represent the distances between planes of electron densities, distances obtained through the MD can be compared with the assignments of the pXRD data, as summarized in Supporting Information, Table S2. To compare the simulated structures to the experimental results, intrafiber distances were measured by means of radial distribution functions (RDF, Supporting Information Figure S14). The RDF maximum representing the length of the molecule, from $5^{\prime}$ carbon to final tail carbon, was found to be 17-18 , which is in accordance with the pXRD analysis (half of the $d$-spacing of the suggested lamellar structure); molecules are in an extended conformation. Additionally, the diameter of the fiber, represented by a maximum in the $\mathrm{RDF}$ between two $5^{\prime}$-carbon atoms of the deoxyribose moiety, was found to be $36 \AA$, again indicating that the SANS measurements represent bundled fibers. To give an insight into the representative distances of the intermolecular hydrogen bonds, the following distances were measured; $0.39 \mathrm{~nm}$ (amide $\mathrm{C}$-amide $\mathrm{N}$ or $0.28 \mathrm{~nm}$ amide $\mathrm{O}-$ amide $\mathrm{N}), 0.31 \mathrm{~nm}$ [base $\mathrm{N}(3)$-amide $\mathrm{N}$ ], and $0.27 \mathrm{~nm}$ [base $\mathrm{O}(2)$-amide $\mathrm{N}]$. These hydrogen bond distances were encountered in different frequencies as presented in Supporting
Information, Figure S15. The broad range of intermolecular hydrogen bond distances, as suggested by the pXRD data, can be supported by the different potential hydrogen bond acceptors proposed by the MD. The aromatic base stacking was found to be fairly flexible with RDF maxima at 3.6 and 5.1 $\AA$ (closest and center-center distance, respectively). Similar lengths of the molecule and distances between atoms of neighboring molecules were also found in the structures that were formed spontaneously from solvated gelator molecules, although in lower intensities because of the more pronounced heterogeneity (Supporting Information, Figure S14). Taken together, these MD data demonstrate the spontaneous formation of fibrous nanostructures with length scales matching those obtained from experimental observations, thus confirming that the proposed fiber structures are reasonable.

\section{CONCLUSIONS}

In the present work, experimental data along with $\mathrm{MD}$ simulation were used together to indicate the self-assembling mechanism and the nanoarchitecture of the fiber of a novel nucleoside (cytosine-based) gelator. The gelator molecules, driven by solvophobic forces, initially assemble into oligomeric structures and then rearrange to orient themselves into a cylindrical fiber with the aliphatic chains toward the core; the nucleobases stacked together and the hydrophilic sugars toward the external surface of the fiber, whereas hydrogen bonds stabilize the structure. A dual environment is formed, displaying hydrophobic cores within the fiber and hydrophilic cavities surrounding them. While some structural elucidation of the self-assembly of nucleoside-based gels exist, this is the first report of the self-assembly of a cytosine-based gelator. Moreover, this work demonstrates that the traditionally less well-established molecular modeling of nucleobase gelators is a powerful approach to deduce information about the self- 
assembly of nucleic acid-based gelators and matches well with experimental data.

The architecture of the present cytidine-based gel presents promising opportunities for the encapsulation of small hydrophobic molecules (e.g., anticancer drugs) as well as the stabilization of hydrophilic biopharmaceuticals (e.g., therapeutic proteins or peptides) in drug delivery applications. The ability to measure, predict, and rationalize nucleobase gelator selfassembly provided by this work opens the way to a rational design of a previously unexplored class of nucleobase gelators based on cytidine for biomedical applications.

\section{EXPERIMENTAL SECTION}

Materials. 2'-Deoxycytidine \{lot \#SLBN6031, 99\% [high-performance liquid chromatography (HPLC)]\} and Nile red (lot 47H3445) were purchased from Sigma Aldrich. The gelator was synthesized according to procedures reported previously. ${ }^{18}$ Solvents (HPLC grade) were obtained from Fischer Scientific. Analysis of the gelator was performed by NMR and liquid chromatography-mass spectrometry (LC-MS) (Supporting Information Figures S16 and S17), and purity was determined as $98 \%$ (LC-MS).

Gel Preparation. In a glass container $(14 \mathrm{~cm}$ diameter), water $(300 \mathrm{~mL})$ was equilibrated at $60{ }^{\circ} \mathrm{C}$. The solvent mixture was prepared by mixing Milli-Q water and ethanol $(80: 20 \mathrm{v} / \mathrm{v} \%)$. $N^{4}$-octanoyl-2'deoxycytidine $(6.00 \pm 0.01 \mathrm{mg})$ was preweighed in $1.5 \mathrm{~mL}$ HPLC vials. The solvent mixture $(1.2 \mathrm{~mL})$ was pipetted into the vials to give a $5 \mathrm{mg} / \mathrm{mL}$ mixture. The vials were placed in the preheated water not in direct contact with the bottom of the container for $2 \mathrm{~min}$. The vials were removed and mildly agitated and left to rest on the bench for 30 s. Consequently, the required volume for the analysis was placed in a suitable container until a transparent gel was produced.

Gels Containing Nile Red. Nile red $(0.32 \mathrm{mg})$ was suspended in a premixed solvent $(20: 80 \mathrm{v} / \mathrm{v} \%$ ethanol/water, $10 \mathrm{~mL})$ and sonicated for $15 \mathrm{~min}$. The gels were prepared as mentioned above using the stock solution of Nile red as the solvent. A freshly made stock solution was prepared for each experiment.

Super Resolution Fluorescent Microscopy. Samples were prepared according to the standard protocol. The warm gelator solution $(5 \mu \mathrm{L})$ was pipetted onto a clean coverslip. Images were recorded with a Zeiss Elyra PS1 with a $561 \mathrm{~nm}$ laser: $0.1 \%$ power100x/ NA1,46 total internal reflection fluorescence (TIRF) objective, with an EMCCD camera in the laser wide-field mode, using TIRF illumination; 35 ms camera exposure time, LBF-561/642 filters.

Fluorescence Spectroscopy. Spectra were recorded at a Cary Eclipse fluorescence spectrophotometer. The spectra were recorded with a scan rate of $30 \mathrm{~nm} / \mathrm{min}$, averaging time $1 \mathrm{~s}$, and data interval 0.5

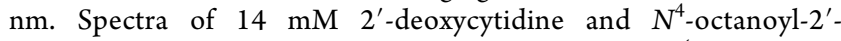
deoxycytidine were recorded at room temperature (in premixed solvent $20: 80 \mathrm{v} / \mathrm{v} \%$ ethanol/water and methanol) after excitation at $326 \mathrm{~nm}$. The excitation slit was set at $2.5 \mathrm{~nm}$ and the emission slit at 5 $\mathrm{nm}$.

CD and Linear Dichroism. Samples of 2 -deoxycytidine were prepared at concentrations of $4.2 \mathrm{mM}$ in methanol and ethanol/water $(20: 80 \mathrm{v} / \mathrm{v} \%)$ in a $1 \mathrm{~mm}$ cuvette, whereas samples of $\mathrm{N}^{4}$-octanoyl-2' deoxycytidine were prepared at $3.5 \mathrm{mM}$ in methanol and $14 \mathrm{mM}$ in ethanol: water $20: 80 \mathrm{v} / \mathrm{v} \%$ in a $1 \mathrm{~mm}$ cuvette and a $0.1 \mathrm{~mm}$ demountable cuvette, respectively. $\mathrm{CD}$ and linear dichroism spectra were recorded simultaneously at a Jasco J-815 instrument. The acquisition parameters were as follows: bandwidth $2 \mathrm{~nm}$, data pitch 0.2 $\mathrm{nm}$, scanning speed $100 \mathrm{~nm} / \mathrm{min}$, and each spectrum was the product of averaging four accumulations.

Fluorescence Temperature Experiment. Spectra of the gels (5 $\mathrm{mg} / \mathrm{mL}$ in premixed solvent $20: 80 \mathrm{v} / \mathrm{v} \%$ ethanol/water) were recorded at $25,30,35,40,45,50,55,60,65$, and $70{ }^{\circ} \mathrm{C}$, with $326 \mathrm{~nm}$ excitation and $382 \mathrm{~nm}$ emission wavelengths. The excitation slit was set at $2.5 \mathrm{~nm}$ and the emission slit at $5 \mathrm{~nm}$. Temperature experiments of the gels after the incorporation of the Nile red $(5 \mathrm{mg} / \mathrm{mL}$ in premixed stock solution $20: 80 \mathrm{v} / \mathrm{v} \%$ ethanol/water) were conducted at the same temperatures as before with $540 \mathrm{~nm}$ excitation and $625 \mathrm{~nm}$ emission wavelengths. The excitation slit was set at $5 \mathrm{~nm}$ and the emission slit at $5 \mathrm{~nm}$.

An excitation spectrum was recorded for the gels $(5 \mathrm{mg} / \mathrm{mL}$ in premixed solvent $20: 80 \mathrm{v} / \mathrm{v} \%$ ethanol/water), and the emission wavelength was set at $382 \mathrm{~nm}$. The excitation slit was set at $2.5 \mathrm{~nm}$ and the emission slit at $5 \mathrm{~nm}$.

Kinetics Experiment. The gel was prepared according to the standard protocol and immediately transferred into the sample holder to record the fluorescent spectrum. Data points were collected for 40 $\mathrm{min}$. For the gel, the excitation slit was set at $2.5 \mathrm{~nm}$ and the emission slit at $5 \mathrm{~nm}$, and the excitation wavelength was $326 \mathrm{~nm}$ and the emission $382 \mathrm{~nm}$. For the gel after the incorporation of the Nile red, the excitation slit was set at $5 \mathrm{~nm}$ and the emission slit at $5 \mathrm{~nm}$, and the excitation wavelength was $540 \mathrm{~nm}$ and the emission $625 \mathrm{~nm}$, averaging time $0.0125 \mathrm{~ns}$.

Powder X-ray Diffraction. Gels were prepared according to the general preparation method mentioned before and left to fully dry at room temperature. $\mathrm{pXRD}$ patterns on the dry gel were collected over the $2 \theta$ range of $3-40^{\circ}$ on a PANalytical X'pert diffractometer using $\mathrm{Cu}$ $\mathrm{K} \alpha \alpha_{1}$ radiation $(\lambda=1.5406 \AA)$.

MD Simulation. MD simulation was performed using the GROMACS 4.6.7 package. ${ }^{55}$ Force-field parameters for the cytidine derivative were based on a combination of the $2^{\prime}$-deoxycytidine and aliphatic tail parameters of the GROMOS54a8 force field. ${ }^{56,57}$ After a short energy minimization, simulations of a pre-ordered fiber $(50 \mathrm{~ns})$ or $50 / 150$ randomly dispersed amphiphiles ( $150 \mathrm{~ns}$ ) in a periodic box of $10 \times 10 \times 10 \mathrm{~nm}$ filled with a pre-equilibrated simple-point-charge water/ethanol mixture ${ }^{58}$ were run in the NPT ensemble with time steps of $1 \mathrm{fs}$. Bonds involving hydrogens were constrained using the LINCS algorithm, ${ }^{59}$ except from water bonds which were constrained using the more efficient SETTLE algorithm. ${ }^{60,61}$ Center of mass motion of the system was removed every 100 steps. Temperature was kept at $298 \mathrm{~K}$ using the velocity-rescaling thermostat ${ }^{61}\left(\tau_{\mathrm{T}}=1.0 \mathrm{ps}\right)$ and pressure at 1.0 bar using the Berendsen barostat ${ }^{62}\left(\tau_{\mathrm{p}}=1.5 \mathrm{ps}\right)$. van der Waals and electrostatic forces were cut off at $1.4 \mathrm{~nm}$ using the Verlet list scheme; long-range electrostatic interactions were treated using a Barker-Watts reaction field with $\varepsilon_{\mathrm{RF}}=62$. Visualization of the simulations was done using the VMD program v. 1.9.3. ${ }^{63} \mathrm{RDF}$ sere calculated on the final one-third of the simulations using the GROMACS package with a bin width of 0.002 (see the Supporting Information for further details).

NMR Experiment. To prepare a sample for analysis by NMR spectroscopy, $4 \mathrm{mg}$ of $\mathrm{N}^{4}$-octanoyl-2'-deoxycytidine was weighed into a $1.2 \mathrm{~mL}$ HPLC vial. A solution of $80 \% \mathrm{D}_{2} \mathrm{O} / 20 \%$ ethanol- $d_{6}$ was then added to the vial to create a $5 \mathrm{mg} / \mathrm{mL}$ mixture of $N^{4}$-octanoyl- $2^{\prime}$ deoxycytidine. The vial was then placed in a water bath at $60{ }^{\circ} \mathrm{C}$ for 2 $\mathrm{min}$, whereupon the vial was removed from the bath, gently shaken, and stood for $30 \mathrm{~s}$. The solution was then injected into a preheated 5 mm NMR tube using a preheated $1 \mathrm{~mL}$ polypropylene syringe fitted with a $200 \mu \mathrm{L}$ polypropylene pipette tip. The NMR tube had been preheated by placing it in the water bath. The syringe was preheated by placing it in a $10 \mathrm{~mm}$ NMR tube which was immersed in the bath. A $2 \mathrm{~mm}$ (outer diameter) capillary (New Era Enterprises, New Jersey, USA) containing $20 \mathrm{mM}$ 3-(trimethylsilyl)propionic-2,2,3,3- $d_{4}$ acid sodium salt (TSP) in $\mathrm{D}_{2} \mathrm{O}$ was then inserted into the sample when still liquid. A clear gel formed in the tube within $10 \mathrm{~min}$. After $1 \mathrm{~h}$ had elapsed, the sample was transferred to the spectrometer for analysis.

NMR experiments were performed on a Bruker AVANCE II 400 $\mathrm{MHz}$ wide bore spectrometer operating at $400.20 \mathrm{MHz}$ for ${ }^{1} \mathrm{H}$. TSP $(0$ $\mathrm{ppm}$ ) was used a reference for all spectra. The sample was heated in 5 ${ }^{\circ} \mathrm{C}$ increments, with a $7 \mathrm{~min}$ equilibration time at each temperature prior to acquiring NMR data. ${ }^{1} \mathrm{H}$ integrals were measured from spectra obtained in four scans using a $30^{\circ}$ excitation pulse, a relaxation delay of $40 \mathrm{~s}$, and a signal acquisition time of $4 \mathrm{~s}$. The total acquisition time was $2 \mathrm{~min}$ and $56 \mathrm{~s}$. STD spectra were acquired using a $90^{\circ}$ excitation pulse and a signal acquisition time of $4 \mathrm{~s}$. The relaxation delay was set at 8.1 $\mathrm{s}$ with presaturation applied during the last second at $-5 \mathrm{ppm}$ (on resonance) and at $-250 \mathrm{ppm}$ (off resonance). Presaturation was delivered using 20 Gaussian pulses of $50 \mathrm{~ms}$ duration and peak powers 
of $380 \mathrm{~Hz}$. The separation between the pulses was $1 \mathrm{~ms}$. On- and offresonance spectra were acquired alternately in four scans at each frequency. Eight dummy scans were acquired prior to signal acquisition. The total acquisition time for STD spectra was thus 3 $\min 16 \mathrm{~s}$.

\section{ASSOCIATED CONTENT}

\section{S Supporting Information}

The Supporting Information is available free of charge on the ACS Publications website at DOI: 10.1021/acs.langmuir.8b00646.

Atomic force microscopy and TEM images of the gel showing the nanoarchitecture, analysis of the fluorescence properties of the gelator, $\mathrm{CD}$ and linear dichroism data of the gel formation, investigations of the variability in the molecular arrangement of the stacked nucleobases, the effect of temperature over time in the self-assembly, additional information of the SANS data and the molecular modeling data as well as structural characterization of the gelator (PDF)

\section{AUTHOR INFORMATION}

\section{Corresponding Authors}

*E-mail: pazmem@exmail.nottingham.ac.uk (M.M.).

*E-mail: pazmz@exmail.nottingham.ac.uk (M.Z.).

\section{ORCID}

Maria G. F. Angelerou: 0000-0001-7191-959X

Pim W. J. M. Frederix: 0000-0002-6892-5611

Matthew Wallace: 0000-0002-5751-1827

Dave J. Adams: 0000-0002-3176-1350

Mischa Zelzer: 0000-0002-6086-2206

\section{Author Contributions}

The manuscript was written through contributions of all authors. M.G.F.A., P.W.J.M.F., M.W., and B.Y. conducted the experimental work. M.Z., M.M., A.R., and D.J.A. contributed to the design, data interpretation, and preparation of the manuscript. All authors have given approval to the final version of the manuscript.

\section{Notes}

The authors declare no competing financial interest.

\section{ACKNOWLEDGMENTS}

This work was support by the EPSRC funded CDT in Targeted Therapeutics grant EP/L01646X and the Leverhulme Trust Research Project Grant (RPG-2016-199). DJA thanks the EPSRC for a Fellowship (EP/L021978/1). We would like to thank Dr Stephen King for help with the SANS experiment at the ISIS Neutron Source (Project RP1710066). P.W.J.M.F. acknowledges support from the Netherlands Organisation for Scientific Research (Veni, 722.015.005). The authors would like to acknowledge Dr Markus Robert, SLIM facility, University of Nottingham for the acquisition of the Superresolution Fluorescence Microscopy images, funded by the BBRSC (reference no. BB/L013827/1). We would also like to thank Rebeca Obero Otenza (University of Nottingham) for acquiring the LC-MS data and Michael C. Nolan (University of Glasgow) for collecting the SANS data. M.W. thanks Unilever for a Case Award and the EPSRC for funding a DTA. M.W. thanks the Royal Commission for the Exhibition of 1851 for a Research Fellowship. The NMR data (Figure 4) was collected at the University of Liverpool using spectrometers purchased/upgraded with EPSRC funding (EP/C005643/1 and EP/K039687/1). Support from Mike Fay at the Nottingham Nanotechnology and Nanoscience Centre (NNNC) for the TEM Imaging is gratefully acknowledged.

\section{REFERENCES}

(1) Adler-Abramovich, L.; Gazit, E. The physical properties of supramolecular peptide assemblies: from building block association to technological applications. Chem. Soc. Rev. 2014, 43, 6881-6893.

(2) Busseron, E.; Ruff, Y.; Moulin, E.; Giuseppone, N. Supramolecular self-assemblies as functional nanomaterials. Nanoscale 2013, 5, 7098-7140.

(3) Skilling, K. J.; Citossi, F.; Bradshaw, T. D.; Ashford, M.; Kellam, B.; Marlow, M. Insights into low molecular mass organic gelators: a focus on drug delivery and tissue engineering applications. Soft Matter 2014, 10, 237-256.

(4) Ramin, M. A.; Sindhu, K. R.; Appavoo, A.; Oumzil, K.; Grinstaff, M. W.; Chassande, O.; Barthélémy, P. Cation Tuning of Supramolecular Gel Properties: A New Paradigm for Sustained Drug Delivery. Adv. Mater. 2017, 29, 1605227.

(5) Nuthanakanti, A.; Srivatsan, S. G. Hierarchical self-assembly of switchable nucleolipid supramolecular gels based on environmentallysensitive fluorescent nucleoside analogs. Nanoscale 2016, 8, 36073619.

(6) Nuthanakanti, A.; Srivatsan, S. G. Surface-tuned and metal ionresponsive supramolecular gels based on nucleolipids. ACS Appl. Mater. Interfaces 2017, 8, 3607.

(7) Godeau, G.; Bernard, J.; Staedel, C.; Barthélémy, P. Glycosylnucleoside-lipid based supramolecular assembly as a nanostructured material with nucleic acid delivery capabilities. Chem. Commun. 2009, 5127-5129.

(8) Johnson, E. K.; Adams, D. J.; Cameron, P. J. Peptide based low molecular weight gelators. J. Mater. Chem. 2011, 21, 2024-2027.

(9) Chen, L.; Morris, K.; Laybourn, A.; Elias, D.; Hicks, M. R.; Rodger, A.; Serpell, L.; Adams, D. J. Self-assembly mechanism for a naphthalene- dipeptide leading to hydrogelation. Langmuir 2009, 26, $5232-5242$.

(10) Peters, G. M.; Davis, J. T. Supramolecular gels made from nucleobase, nucleoside and nucleotide analogs. Chem. Soc. Rev. 2016, 45, 3188-3206.

(11) Baillet, J.; Desvergnes, V.; Hamoud, A.; Latxague, L.; Barthélémy, P. Lipid and Nucleic Acid Chemistries: Combining the Best of Both Worlds to Construct Advanced Biomaterials. Adv. Mater. 2018, 30, 1705078.

(12) Ramin, M. A.; Latxague, L.; Sindhu, K. R.; Chassande, O.; Barthélémy, P. Low molecular weight hydrogels derived from urea based-bolaamphiphiles as new injectable biomaterials. Biomaterials 2017, 145, 72-80.

(13) Kaplan, J. A.; Barthélémy, P.; Grinstaff, M. W. Self-assembled nanofiber hydrogels for mechanoresponsive therapeutic anti-TNF $\alpha$ antibody delivery. Chem. Commun. 2016, 52, 5860-5863.

(14) Allain, V.; Bourgaux, C.; Couvreur, P. Self-assembled nucleolipids: from supramolecular structure to soft nucleic acid and drug delivery devices. Nucleic Acids Res. 2011, 40, 1891-1903.

(15) Plank, T. N.; Davis, J. T. AG 4· K+ hydrogel that self-destructs. Chem. Commun. 2016, 52, 5037-5040.

(16) Sreenivasachary, N.; Lehn, J.-M. Structural Selection in GQuartet-Based Hydrogels and Controlled Release of Bioactive Molecules. Chem.-Asian J. 2008, 3, 134-139.

(17) Maisani, M.; Ziane, S.; Ehret, C.; Levesque, L.; Siadous, R.; Le Meins, J.-F.; Chevallier, P.; Barthélémy, P.; De Oliveira, H.; Amédée, J. A new composite hydrogel combining the biological properties of collagen with the mechanical properties of a supramolecular scaffold for bone tissue engineering. J. Tissue Eng. Regener. Med. 2017, 12, No. e1489.

(18) Skilling, K. J.; Kellam, B.; Ashford, M.; Bradshaw, T. D.; Marlow, M. Developing a self-healing supramolecular nucleoside hydrogel. Soft Matter 2016, 12, 8950-8957. 
(19) Angelerou, M. G. F.; Sabri, A.; Creasey, R.; Angelerou, P.; Marlow, M.; Zelzer, M. Surface-directed modulation of supramolecular gel properties. Chem. Commun. 2016, 52, 4298-4300.

(20) Vigier-Carrière, C.; Boulmedais, F.; Schaaf, P.; Jierry, L. Surfaceassisted self-assembly strategies leading to supramolecular hydrogels. Angew. Chem., Int. Ed. 2017, 57, 1448.

(21) Smith, A. M.; Williams, R. J.; Tang, C.; Coppo, P.; Collins, R. F.; Turner, M. L.; Saiani, A.; Ulijn, R. V. Fmoc-Diphenylalanine Self Assembles to a Hydrogel via a Novel Architecture Based on $\pi-\pi$ Interlocked $\beta$-Sheets. Adv. Mater. 2008, 20, 37-41.

(22) Moreau, L.; Barthélémy, P.; El Maataoui, M.; Grinstaff, M. W. Supramolecular assemblies of nucleoside phosphocholine amphiphiles. J. Am. Chem. Soc. 2004, 126, 7533-7539.

(23) Iwaura, R.; Yoshida, K.; Masuda, M.; Yase, K.; Shimizu, T. Spontaneous fiber formation and hydrogelation of nucleotide bolaamphiphiles. Chem. Mater 2002, 14, 3047-3053.

(24) Bogliotti, N.; Ritter, A.; Hebbe, S.; Vasella, A. Oligonucleotide Analogues with Integrated Bases and Backbones. Part 19. Helv. Chim. Acta 2008, 91, 2181-2202.

(25) Banerjee, P.; Pyne, A.; Pal, S.; Karan, C. K.; Sarkar, N. Selfassembling behavior of pyrimidine analogues: Unveiling the factors behind morphological diversity. J. Colloid Interface Sci. 2018, 522, 6373.

(26) Roviello, G. N.; Oliviero, G.; Di Napoli, A.; Borbone, N.; Piccialli, G. Synthesis, self-assembly-behavior and biomolecular recognition properties of thyminyl dipeptides. Arabian J. Chem. 2018, DOI: $10.1016 /$ j.arabjc.2018.02.014.

(27) Skilling, K. J.; Ndungu, A.; Kellam, B.; Ashford, M.; Bradshaw, T. D.; Marlow, M. Gelation properties of self-assembling $\mathrm{N}$-acyl modified cytidine derivatives. J. Mater. Chem. B 2014, 2, 8412-8417.

(28) Thota, N.; Jiang, J. Computational amphiphilic materials for drug delivery. Front. Mater. 2015, 2, 64.

(29) Manandhar, A.; Kang, M.; Chakraborty, K.; Tang, P. K.; Loverde, S. M. Molecular simulations of peptide amphiphiles. Org. Biomol. Chem. 2017, 15, 7993-8005.

(30) Yuan, C.; Li, S.; Zou, Q.; Ren, Y.; Yan, X. Multiscale simulations for understanding the evolution and mechanism of hierarchical peptide self-assembly. Phys. Chem. Chem. Phys. 2017, 19, 23614-23631.

(31) Pan, D.; Tang, C.; Fan, X.; Li, Y.; Yang, X.; Jin, H.; Guan, Z.; Yang, Z.; Zhang, L. Thymidine-based amphiphiles and their bonding to DNA. New J. Chem. 2013, 37, 1122-1127.

(32) Lepeltier, E.; Bourgaux, C.; Rosilio, V.; Poupaert, J. H.; Meneau, F.; Zouhiri, F.; Lepêtre-Mouelhi, S.; Desmaële, D.; Couvreur, P. Selfassembly of squalene-based nucleolipids: relating the chemical structure of the bioconjugates to the architecture of the nanoparticles. Langmuir 2013, 29, 14795-14803.

(33) Raeburn, J.; Adams, D. J. Multicomponent low molecular weight gelators. Chem. Commun. 2015, 51, 5170-5180.

(34) Du, X.; Zhou, J.; Shi, J.; Xu, B. Supramolecular hydrogelators and hydrogels: from soft matter to molecular biomaterials. Chem. Rev. 2015, 115, 13165-13307.

(35) Fichman, G.; Gazit, E. Self-assembly of short peptides to form hydrogels: Design of building blocks, physical properties and technological applications. Acta Biomater. 2014, 10, 1671-1682.

(36) Hughes, M.; Birchall, L. S.; Zuberi, K.; Aitken, L. A.; Debnath, S.; Javid, N.; Ulijn, R. V. Differential supramolecular organisation of Fmoc-dipeptides with hydrophilic terminal amino acid residues by biocatalytic self-assembly. Soft Matter 2012, 8, 11565-11574.

(37) Nordén, B.; Rodger, A.; Dafforn, T. Linear Dichroism and Circular Dichroism; The Royal Society of Chemistry, 2010.

(38) Maitra, U.; Mukhopadhyay, S.; Sarkar, A.; Rao, P.; Indi, S. S. Hydrophobic pockets in a nonpolymeric aqueous gel: observation of such a gelation process by color change. Angew. Chem., Int. Ed. 2001, 40, 2281-2283.

(39) Greenspan, P.; Mayer, E. P.; Fowler, S. D. Nile red: a selective fluorescent stain for intracellular lipid droplets. J. Cell Biol. 1985, 100, 965-973.

(40) Gels, M. Materials with Self-Assembled Fibrillar Networks; Weiss, R. G., Terech, P., Eds.; Springer: Dordrecht, 2006.
(41) Morris, K. L.; Chen, L.; Raeburn, J.; Sellick, O. R.; Cotanda, P.; Paul, A.; Griffiths, P. C.; King, S. M.; O’Reilly, R. K.; Serpell, L. C. Chemically programmed self-sorting of gelator networks. Nat. Commun. 2013, 4, 1480.

(42) Escuder, B.; LLusar, M.; Miravet, J. F. Insight on the NMR study of supramolecular gels and its application to monitor molecular recognition on self-assembled fibers. J. Org. Chem. 2006, 71, 77477752.

(43) Hirst, A. R.; Coates, I. A.; Boucheteau, T. R.; Miravet, J. F.; Escuder, B.; Castelletto, V.; Hamley, I. W.; Smith, D. K. Lowmolecular-weight gelators: elucidating the principles of gelation based on gelator solubility and a cooperative self-assembly model. J. Am. Chem. Soc. 2008, 130, 9113-9121.

(44) Angulo, J.; Enríquez-Navas, P. M.; Nieto, P. M. Ligandreceptor binding affinities from saturation transfer difference (STD) NMR spectroscopy: the binding isotherm of STD initial growth rates. Chem.-Eur. J. 2010, 16, 7803-7812.

(45) Nonappa; Šaman, D.; Kolehmainen, E. Studies on supramolecular gel formation using DOSY NMR. Magn. Reson. Chem. 2015, $53,256-260$.

(46) Duncan, D. C.; Whitten, D. G. 1H NMR Investigation of the Composition, Structure, and Dynamics of Cholesterol- Stilbene Tethered Dyad Organogels. Langmuir 2000, 16, 6445-6452.

(47) Ramalhete, S. M.; Nartowski, K. P.; Sarathchandra, N.; Foster, J. S.; Round, A. N.; Angulo, J.; Lloyd, G. O.; Khimyak, Y. Z. Supramolecular amino acid based hydrogels: probing the contribution of additive molecules using NMR spectroscopy. Chem.-Eur. J. 2017, 23, 8014-8024.

(48) Segarra-Maset, M. D.; Escuder, B.; Miravet, J. F. Selective Interaction of Dopamine with the Self-Assembled Fibrillar Network of a Molecular Hydrogel Revealed by STD-NMR. Chem.-Eur. J. 2015, 21, 13925-13929.

(49) Wallace, M.; Iggo, J. A.; Adams, D. J. Probing the surface chemistry of self-assembled peptide hydrogels using solution-state NMR spectroscopy. Soft Matter 2017, 13, 1716-1727.

(50) Iqbal, S.; Rodríguez-LLansola, F.; Escuder, B.; Miravet, J. F.; Verbruggen, I.; Willem, R. HRMAS $1 \mathrm{H}$ NMR as a tool for the study of supramolecular gels. Soft Matter 2010, 6, 1875-1878.

(51) Raeburn, J.; Chen, L.; Awhida, S.; Deller, R. C.; Vatish, M.; Gibson, M. I.; Adams, D. J. Using molecular rotors to probe gelation. Soft Matter 2015, 11, 3706-3713.

(52) Ryan, D. M.; Anderson, S. B.; Senguen, F. T.; Youngman, R. E.; Nilsson, B. L. Self-assembly and hydrogelation promoted by F 5phenylalanine. Soft Matter 2010, 6, 475-479.

(53) Mears, L. L. E.; Draper, E. R.; Castilla, A. M.; Su, H.; Dietrich, B.; Nolan, M. C.; Smith, G. N.; Doutch, J.; Rogers, S.; Akhtar, R. Drying affects the fiber network in low molecular weight hydrogels. Biomacromolecules 2017, 18, 3531-3540.

(54) Baldelli Bombelli, F.; Berti, D.; Keiderling, U.; Baglioni, P. Giant polymerlike micelles formed by nucleoside-functionalized lipids. $J$. Phys. Chem. B 2002, 106, 11613-11621.

(55) Hess, B.; Kutzner, C.; Van Der Spoel, D.; Lindahl, E. GROMACS 4: algorithms for highly efficient, load-balanced, and scalable molecular simulation. J. Chem. Theory Comput. 2008, 4, 435447.

(56) Reif, M. M.; Hünenberger, P. H.; Oostenbrink, C. New interaction parameters for charged amino acid side chains in the GROMOS force field. J. Chem. Theory Comput. 2012, 8, 3705-3723.

(57) Reif, M. M.; Winger, M.; Oostenbrink, C. Testing of the GROMOS force-field parameter set 54A8: structural properties of electrolyte solutions, lipid bilayers, and proteins. J. Chem. Theory Comput. 2013, 9, 1247-1264.

(58) Berendsen, H. J.; Postma, J. P.; van Gunsteren, W. F.; Hermans, $\mathrm{J}$. Interaction models for water in relation to protein hydration. Intermolecular Forces; Springer, 1981; pp 331-342.

(59) Hess, B. P-LINCS: A parallel linear constraint solver for molecular simulation. J. Chem. Theory Comput. 2008, 4, 116-122. 
(60) Miyamoto, S.; Kollman, P. A. SETTLE: an analytical version of the SHAKE and RATTLE algorithm for rigid water models. J. Comput. Chem. 1992, 13, 952-962.

(61) Bussi, G.; Donadio, D.; Parrinello, M. Canonical sampling through velocity rescaling. J. Chem. Phys. 2007, 126, 014101.

(62) Berendsen, H. J. C.; Postma, J. P. M.; van Gunsteren, W. F.; DiNola, A.; Haak, J. R. Molecular dynamics with coupling to an external bath. J. Chem. Phys. 1984, 81, 3684-3690.

(63) Humphrey, W.; Dalke, A.; Schulten, K. VMD: Visual Molecular Dynamics. J. Mol. Graphics 1996, 14, 33-38. 